\title{
Dynamic Pressure Gradient Model of Axial Piston Pump and Parameters Optimization
}

\author{
Shi Jian, ${ }^{1}$ Li Xin, ${ }^{1}$ and Wang Shaoping ${ }^{1,2}$ \\ ${ }^{1}$ School of Automation and Electrical Engineering, Beihang University, Beijing 100191, China \\ ${ }^{2}$ Science and Technology on Aircraft Control Laboratory, Beihang University, Beijing 100191, China \\ Correspondence should be addressed to Shi Jian; shijian123@sina.com
}

Received 21 October 2013; Revised 5 January 2014; Accepted 6 January 2014; Published 12 February 2014

Academic Editor: Evangelos J. Sapountzakis

Copyright (c) 2014 Shi Jian et al. This is an open access article distributed under the Creative Commons Attribution License, which permits unrestricted use, distribution, and reproduction in any medium, provided the original work is properly cited.

\begin{abstract}
The unsteady pressure gradient can cause flow noise in prepressure rising of piston pump, and the fluid shock comes up due to the large pressure difference of the piston chamber and discharge port in valve plate. The flow fluctuation control is the optimization objective in previous study, which cannot ensure the steady pressure gradient. Our study is to stabilize the pressure gradient in prepressure rising and control the pressure of piston chamber approaching to the pressure in discharge port after prepressure rising. The models for nonoil shock and dynamic pressure of piston chamber in prepressure rising are established. The parameters of prepressure rising angle, cross angle, wrap angle of V-groove, vertex angle of V-groove, and opening angle of V-groove were optimized, based on which the pressure of the piston chamber approached the pressure in discharge port after prepressure rising, and the pressure gradient is more steady compared to the original parameters. The max pressure gradient decreased by $70.8 \%$ and the flow fluctuation declined by $21.4 \%$, which showed the effectivness of optimization.
\end{abstract}

\section{Introduction}

Axial piston pump is widely used in hydraulic system due to the advantages of high pressure, compact structure, high efficiency, and flow regulation. The flow fluctuation is the inherent characteristic based on the working principle. Flow fluctuation results in pressure fluctuation which affects the pipe vibration [1]. The flow fluctuation control is a significant method to restrain the pipe vibration and flow noise.

Due to the large pressure difference of the oil in piston chamber and discharge port, the oil impulse exists when one piston moves to the discharge port. The pressure of the piston chamber should be increased to the pressure of the discharge port, and then there would be no oil impulse. There are two methods of pressure increasing in advance. (1) The cross angle is set on the valve plate which is nonsymmetrical, and the pressure of the piston chamber can be increased more than that of the symmetrical valve plate; however, the angle of the discharge port is smaller than that of the symmetrical valve plate, which would affect the flow output. (2) Designing an oil way on the valve plate of the prepressure rising region, then the oil can flow backward to the piston chamber. The V-groove and damping hole are two common structures.

References [2-6] analyzed the pressure distribution on the valve plate, which shows that the max pressure peak exists near the discharge port of the valve plate. On the other hand, the oil pressure in discharge port is much larger than in the suction port, and the maximum flow fluctuation is determined by the valve plate structure near the discharge port.

The structure optimization of valve plate is the main method to control the flow fluctuation of the pump. Reference [7] analyzed the effect of the structure of the valve plate on the pressure fluctuation characteristic and presented a new structure with the damping hole and V-groove. Reference [8] set the dynamic pressure model of the piston chamber and optimized the cross angle and precompressing angle. Reference [9] optimized the damping hole by using rotating vector method, and the value of the flow fluctuation reduced by $40 \%$. Reference [10] analyzed three different types (a constant area slot geometry, a linearly varying slot geometry, and a quadratically varying slot geometry) of the valve plate, and the results showed that the constant area slot had the 
advantage of minimizing the required discharge area of the slot, the linearly varying slot had the shortest slot length, and the quadratically varying slot showed the worst performance. Reference [11] designed a new adaptive valve plate to control the flow fluctuation, but the reliability is low. Reference [12] analyzed the effect of the cross angle on the valve plate to the flow fluctuation, and the flow fluctuation decreased based on the optimization of the cross angle.

It is an effective method that the damping groove (hole) and cross angle are optimized to control the flow fluctuation. In previous study, only the parts of the valve plate were optimized; however, the entire structure of the valve plate should be considered. Additionally, the previous optimal objective is to control the flow fluctuation. The pressure gradient in prepressure rising is another significant factor because the flow noise is proportional to the pressure gradient, so the optimizing objective should consider the comprehensive factor of the flow fluctuation and pressure gradient in prepressure rising.

In this paper, Section 2 illustrated the optimal models of cross angle and close angle of pressure rising which can avoid the oil shock. Section 3 showed the dynamic pressure gradient model of piston in a period. Section 4 showed the pressure gradient variation with different cross angles, close angles, wrap angles of $\mathrm{V}$-groove, opening angles of $\mathrm{V}$-groove, and vertical angles of V-groove. Section 5 illustrated the optimization of five angles using particle swarm optimization (PSO) algorithm to make the pressure gradient in prepressure rising steadiness.

\section{The Model for Nonoil Shock}

The oil is compressed after piston passes the upper point. Generally, the pressure in piston chamber is lower than that of the discharge port before the piston chamber moves to the $\mathrm{V}$ groove or damping hole, so the oil in the discharge port would flow back to the piston chamber. It results in the variation of the pressure in piston chamber. Figure 1 shows the cutaway view of the piston pump and the structure of the valve plate.

Suppose the pressure in piston chamber $P$ when the piston chamber is on the upper point, the pressure in discharge port $P_{s}$, so the variation of the pressure in piston chamber $\Delta P$ in prepressure rising is

$$
\Delta P=P_{s}-P \text {. }
$$

The increment equation is

$$
\begin{gathered}
\Delta P=-E \frac{\Delta V_{1}+\Delta V_{2}}{V_{0}} \\
\Delta V_{1}=\frac{1}{\omega} \int_{0}^{\varphi_{\Delta}} Q \mathrm{~d} \varphi \\
\Delta V_{2}=-A R \operatorname{tg} \gamma\left[\cos \left(\Delta \varphi-\frac{\varphi_{0}}{2}\right)-\cos \left(\Delta \varphi-\frac{\varphi_{0}}{2}+\varphi_{0}\right)\right] \\
=-2 A R \operatorname{tg} \gamma \sin \Delta \varphi \sin \frac{\varphi_{0}}{2}
\end{gathered}
$$

where $E$ is the volume modulus of the oil, $\Delta V_{1}$ is the volume from the discharge port to the piston chamber in prepressure rising, $\Delta V_{2}$ is the variation in compressing process of the oil in piston chamber, $V_{0}$ is the initial volume of piston chamber at upper point, $\omega$ is the rotational speed of the piston pump, $\varphi_{\Delta}$ is the wrap angle of the $\mathrm{V}$-groove, $\Delta \varphi$ is the cross angle of the valve plate, $A$ is the area of the piston, $R$ is the radius of the distribution circle of the pistons, $\gamma$ is the swashplate angle, and $\varphi$ is the variable angle of the piston moves.

Set $K_{1}=\Delta V_{1} /\left(\Delta V_{1}+\left|\Delta V_{2}\right|\right)$; then,

$$
\Delta V_{1}=\frac{K_{1}}{1-K_{1}}\left|\Delta V_{2}\right|
$$

Substitute (4) and (5) to (2), then

$$
\Delta P=E \frac{2 A R \sin \Delta \varphi \sin \left(\varphi_{0} / 2\right)\left(\left(K_{1} /\left(1-K_{1}\right)\right) \operatorname{tg} \gamma_{s}+\operatorname{tg} \gamma\right)}{V_{0 s}+A R\left[\operatorname{tg} \gamma_{s}+\operatorname{tg} \gamma \cos \left(\Delta \varphi-\left(\varphi_{0} / 2\right)\right)\right]},
$$

where $V_{0 s}$ is the volume of dead zone in piston chamber and $\gamma_{s}$ is the swashplate angle under full flow of the piston pump.

Generally, the angle of $\Delta \varphi-\varphi_{0} / 2$ is no more than $3^{\circ}$, so $\cos \left(\Delta \varphi-\varphi_{0} / 2\right) \approx 1$; then,

$$
\Delta P=E \frac{\left(\left(K_{1} /\left(1-K_{1}\right)\right)+\left(\operatorname{tg} \gamma / \operatorname{tg} \gamma_{s}\right)\right) \sin \Delta \varphi \sin \left(\varphi_{0} / 2\right)}{K_{A}+(1 / 2)\left(1+\left(\operatorname{tg} \gamma / \operatorname{tg} \gamma_{s}\right)\right)},
$$

where $K_{A}=V_{0 s} / 2 A R \operatorname{tg} \gamma_{s}$.

Reference [13] illustrated the equations under non-oil shock condition.

(1) Full flow $\left(\gamma=\gamma_{s}\right)$ : the pressure of the piston chamber increases based on the volume compressing of piston chamber and oil flowing back from the discharge port to the piston chamber:

$$
\Delta P=E \frac{\left(1 /\left(1-K_{1}\right)\right) \sin \Delta \varphi \sin \left(\varphi_{0} / 2\right)}{K_{A}+1} .
$$

(2) Zero flow $(\gamma=0)$ : the pressure of the piston chamber increases only based on the oil flowing back from the discharge port to the piston chamber:

$$
\Delta P=E \frac{\left(K_{1} /\left(1-K_{1}\right)\right) \sin \Delta \varphi \sin \left(\varphi_{0} / 2\right)}{K_{A}+(1 / 2)} .
$$

There is no oil shock under zero flow condition, so if (8) equals (9), there is no oil shock under full flow condition; then,

$$
K_{1}=\frac{K_{A}+(1 / 2)}{K_{A}+1} .
$$

Equation (8) can be changed to (11):

$$
\sin \Delta \varphi \sin \frac{\varphi_{0}}{2}=\frac{\Delta P}{E}\left(K_{A}+1\right)\left(1-K_{1}\right) .
$$

Substitute (10) to (11); then,

$$
\sin \Delta \varphi \sin \frac{\varphi_{0}}{2}=\frac{1}{2} \frac{\Delta P}{E} .
$$




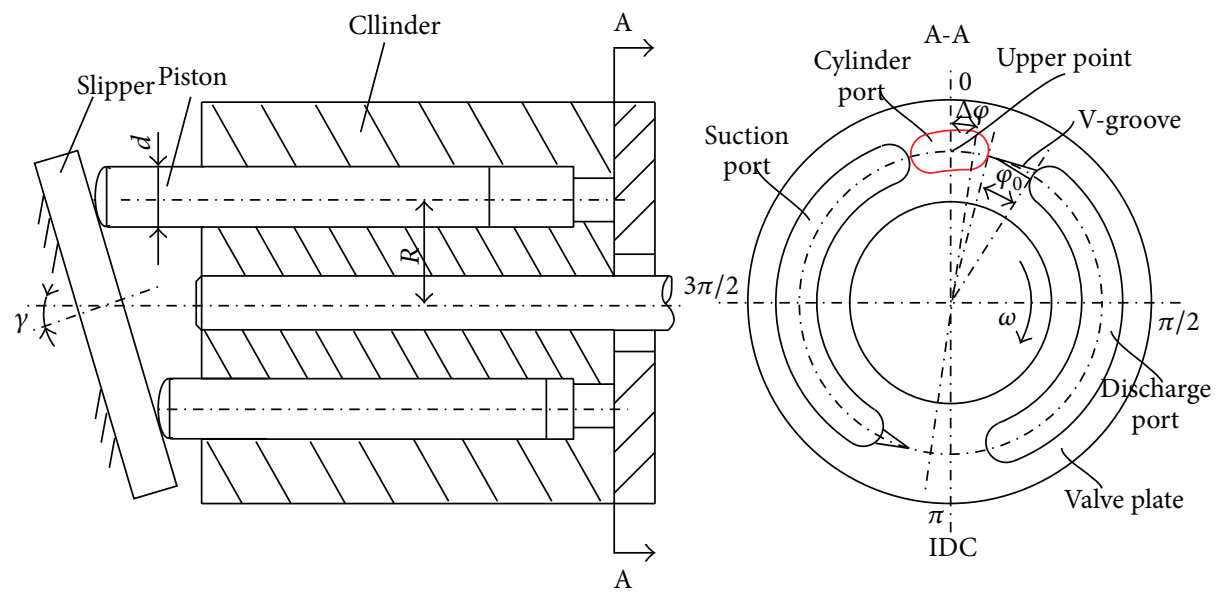

FIGURE 1: Cutaway view of the piston pump and the structure of the valve plate.
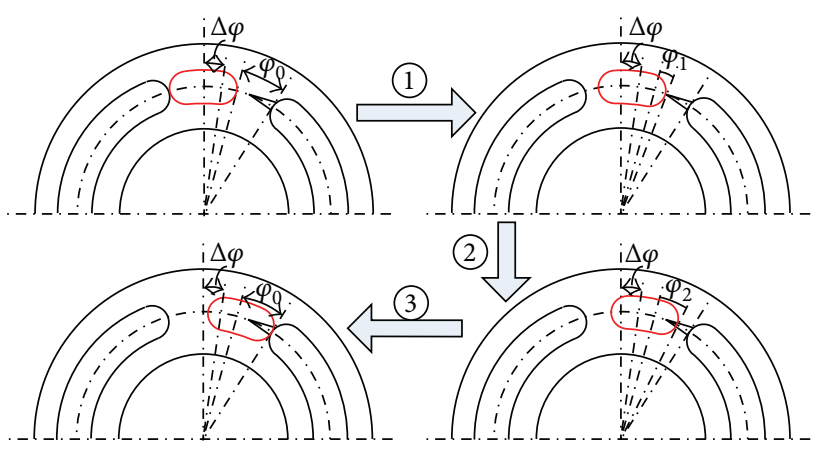

FIgURE 2: Three stages of piston chamber moves.

As we can see from (12), the oil shock can be avoided under the relation between $\Delta \varphi$ and $\varphi_{0}$. Additionally, (12) cannot guarantee the steady pressure gradient, so the dynamic pressure gradient of the piston chamber should be obtained, based on which the parameters of the valve plate can be optimized to get the steady pressure gradient.

\section{Dynamic Pressure Gradient Model of Piston Chamber}

There are three stages when the piston chamber moves out from the suction port to the discharge port.

As we can see from Figure 2, the three stages of piston chamber are as follows.

Stage 1. Prepressure rising (the volume compressing of piston chamber); the piston chamber moves out from the suction port to the vertex of $\mathrm{V}$-groove.

Stage 2. Prepressure rising (the volume compressing of piston chamber and the oil flow back to the piston chamber); the piston chamber moves to the location when the pressure of the piston chamber equals the pressure of the discharge port.
Stage 3. Oil discharge of the piston chamber; the piston chamber moves to the top location of the discharge port:

$$
\begin{gathered}
\frac{d P}{d \varphi}=E \frac{A \omega R \tan \gamma \sin \left(\Delta \varphi-\left(\varphi_{0} / 2\right)+\varphi\right)-k_{l} P}{\omega V_{0}} \text { Stage 1, } \\
\frac{d P}{d \varphi}=E \frac{C_{d} A_{0} \sqrt{\left(2\left(P_{s}-P\right) / \rho\right)}-k_{l} P}{\omega V_{0}} \\
+E \frac{A \omega R \tan \gamma \sin \left(\Delta \varphi-\left(\varphi_{0} / 2\right)+\varphi\right)}{\omega V_{0}} \quad \text { Stage 2, }
\end{gathered}
$$

$$
\begin{aligned}
\frac{d P}{d \varphi}= & E \frac{-C_{d} A_{0} \sqrt{\left(2\left(P-P_{s}\right) / \rho\right)}-k_{l} P}{\omega V_{0}} \\
& +E \frac{A \omega R \tan \gamma \sin \left(\Delta \varphi-\left(\varphi_{0} / 2\right)+\varphi\right)}{\omega V_{0}} \quad \text { Stage 3, } \\
& A_{0}=R^{2} \varphi^{2} \tan ^{2} \theta_{1} \tan \frac{\theta_{2}}{2} \quad 0 \leq \phi \leq \varphi_{1},
\end{aligned}
$$

where $P$ is the pressure of the piston chamber, $\varphi$ is the angle of the piston chamber moves, $E$ is the volume modulus of the oil, $A$ is the area of the piston, $\omega$ is the rotational speed of the piston pump, $\gamma$ is the swashplate angle, $\Delta \varphi$ is the cross angle of the valve plate, $\varphi_{0}$ is the close angle, $k_{l}$ is the coefficient of leakage, $C_{d}$ is the flow coefficient, $A_{0}$ is the oil-transit area of the $\mathrm{V}$-groove, $P_{s}$ is the pressure of the discharge port, $V_{0}$ is the initial volume of piston chamber at upper point, $\theta_{1}$ is the opening angle of the $\mathrm{V}$-groove, and $\theta_{2}$ is the vertical angle of the V-groove.

\section{Simulation of the Dynamic Pressure of the Piston Chamber}

The original parameters of the piston pump are shown in Table 1. 
TABLE 1: The original parameters of the valve plate and piston pump.

\begin{tabular}{lc}
\hline Variable & Valve \\
\hline Close angle $\varphi_{0}$ & $12^{\circ}$ \\
Cross angle $\Delta \varphi$ & $5^{\circ}$ \\
Wrap angle of the V-groove $\varphi_{\Delta}$ & $12^{\circ}$ \\
Opening angle of the V-groove $\theta_{1}$ & $30^{\circ}$ \\
Vertex angle of the V-groove $\theta_{2}$ & $90^{\circ}$ \\
Radius of the distribution circle of pistons $R$ & $16.85 \mathrm{~mm}$ \\
Piston number & 9 \\
Rotational speed of the Pump $\omega$ & $7800 \mathrm{r} / \mathrm{min}$ \\
Swashplate angle $\gamma_{s}$ & $14.5^{\circ}$ \\
\hline
\end{tabular}

According to (13) and Table 1, the pressure variation of the piston chamber is shown in Figure 3.

As we can see Figure 3, the pressure variation starts from Stage 2 due to $\varphi_{0}=\varphi_{\Delta}$, the pressure rising is due to the volume compressing of piston chamber and the oil flow back to the piston chamber, and the pressure gradient increases with the rotational angle increasing. The piston chamber starts to discharge oil when the pressure increases to $20 \mathrm{MPa}$, and the pressure gradient begins to decline. The pressure is $26 \mathrm{MPa}$ when the rotation angle is $12^{\circ}$, so there is an oil shock when the piston chamber moves to the discharge port.

The close angle $\varphi_{0}$, cross angle $\Delta \varphi$, wrap angle of $\mathrm{V}$-groove $\varphi_{\Delta}$, opening angle of $\mathrm{V}$-groove $\theta_{1}$, and vertex angle $\theta_{2}$ play important roles in the pressure of the piston chamber, and the five angles should be analyzed.

4.1. The Effect of Close Angle on Pressure Gradient of the Piston Chamber. As we can see from Figure 4, there are about 3 kinds of $\varphi_{0}$ for volume expansion of the piston chamber when the close angle is $15^{\circ}$, and the pressure of the piston chamber decreases from $2 \mathrm{MPa}$ to $0.35 \mathrm{MPa}$. The piston chamber starts to compress when it leaves the suction port when the close angle is $10^{\circ}$ or $12^{\circ}$. The oil is discharged from the $\mathrm{V}$-groove when the pressure of the piston chamber is $20 \mathrm{MPa}$; then, the pressure gradient began to decrease. The turning point is $8.48^{\circ}, 9.05^{\circ}$, and $10.31^{\circ}$ for $\varphi_{0}=10^{\circ}, \varphi_{0}=12^{\circ}$, and $\varphi_{0}=15^{\circ}$.

\subsection{The Effect of Angle of the V-Groove on Pressure Gradient} of the Piston Chamber. Figure 5 shows pressure variation of the piston chamber with different open angles of $\mathrm{V}$-groove when $\varphi_{0}=12^{\circ}$. The pressure gradient continues to increase when $\theta_{1}=20^{\circ}, \theta_{2}=60^{\circ}$, and there is little oil passed from the $\mathrm{V}$-groove. The oil flowing back to the piston chamber increases when the open angle of the $\mathrm{V}$-groove increases, and the pressure gradient of the piston chamber increases faster. However, the pressure gradient of the piston chamber with lager open angle has rapid decrease when the pressure of the piston chamber is more than $20 \mathrm{MPa}$.

\subsection{The Effect of Cross Angle on Pressure Gradient of the Piston} Chamber. As we can see from Figure 6, the compressing area increases along with the cross angle increasing, so the pressure also increases faster with the cross angle increasing.

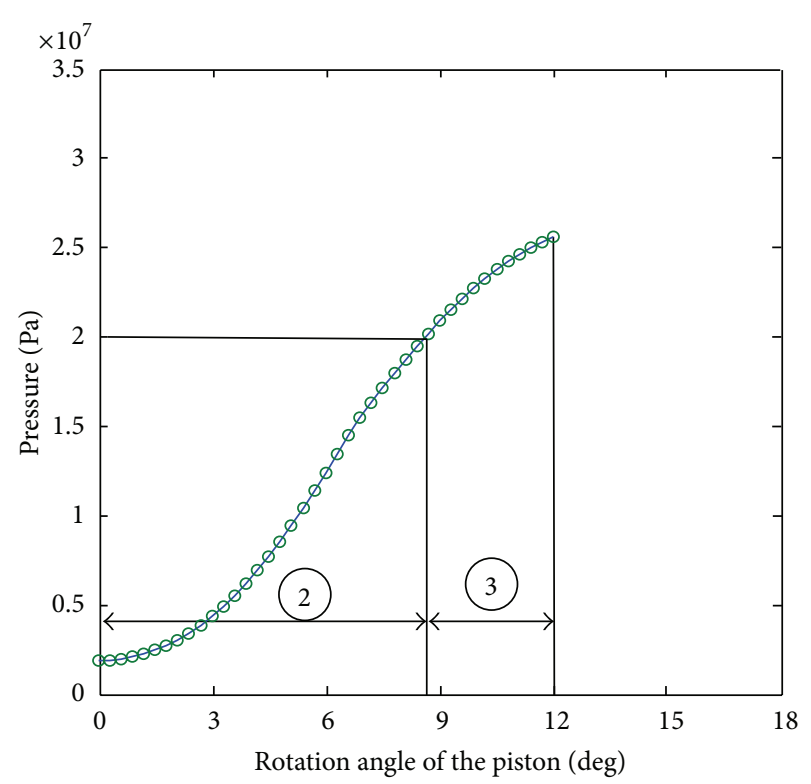

Figure 3: Pressure variation of the piston chamber.

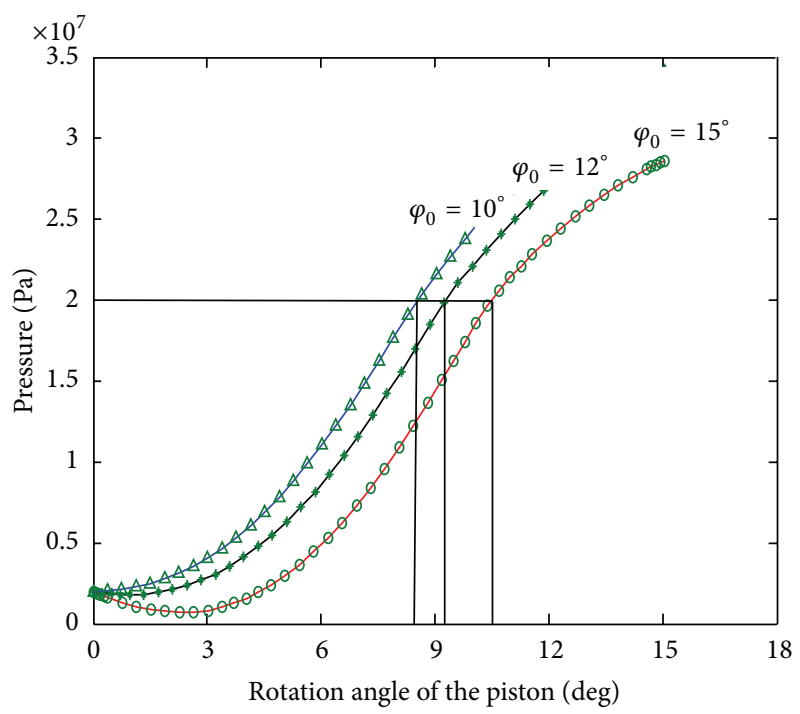

FIGURE 4: Pressure variation of the piston chamber.

In contrast, the volume of piston chamber would increase, and the pressure would decrease in initial phase when the cross angle is small.

4.4. The Effect of Wrap Angle of the V-Groove on Pressure Gradient of the Piston Chamber. As we can see from Figure 7, the pressure gradient when $\varphi_{\Delta}=14^{\circ}$ is the largest of the three angles in initial phase because the oil flows back to the piston chamber when the piston just moves out from the suction port; in contrast, the pressure gradient is the least when $\varphi_{\Delta}=8^{\circ}$ at the initial phase. The pressure gradient decreases the fastest when $\varphi_{\Delta}=14^{\circ}$ after the pressure of the piston chamber exceeds $20 \mathrm{MPa}$ due to the largest area of oil passing from the $\mathrm{V}$-groove; in contrast, the pressure gradient 


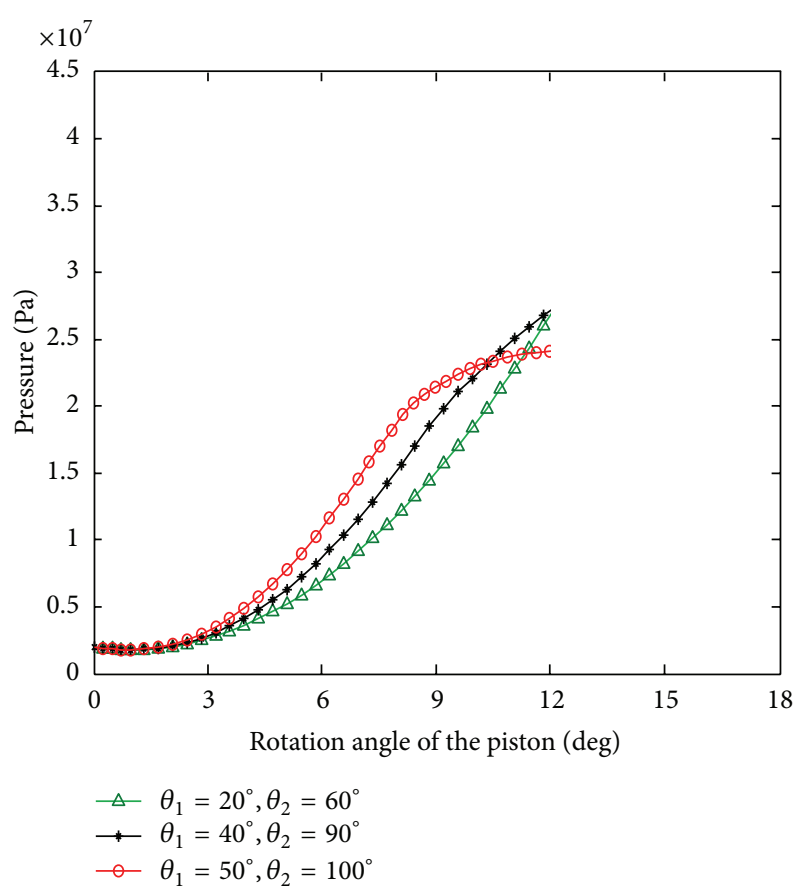

Figure 5: Pressure variation of the piston chamber.

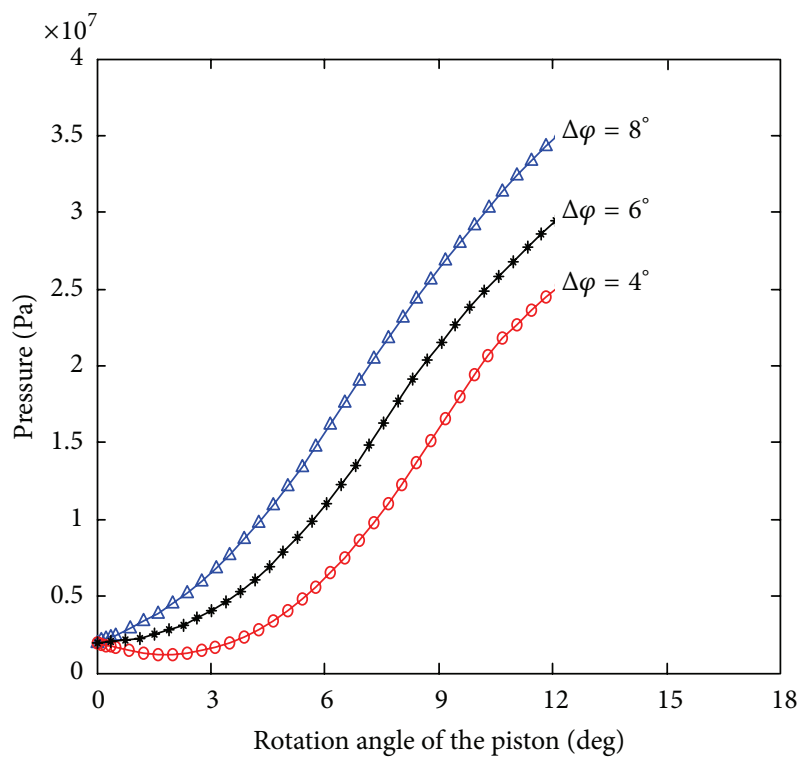

FIgURE 6: Pressure variation of the piston chamber.

when $\varphi_{\Delta}=8^{\circ}$ is the largest after the pressure of the piston chamber exceeds $20 \mathrm{MPa}$.

\section{Parameter Optimization of the Valve Plate Based on the Steady Dynamic Pressure Gradient}

5.1. Ideal steady Dynamic Pressure Gradient. As we can see from Figure 3, the pressure is $26 \mathrm{MPa}$, which is much more than the pressure in the discharge part, so there exists a large

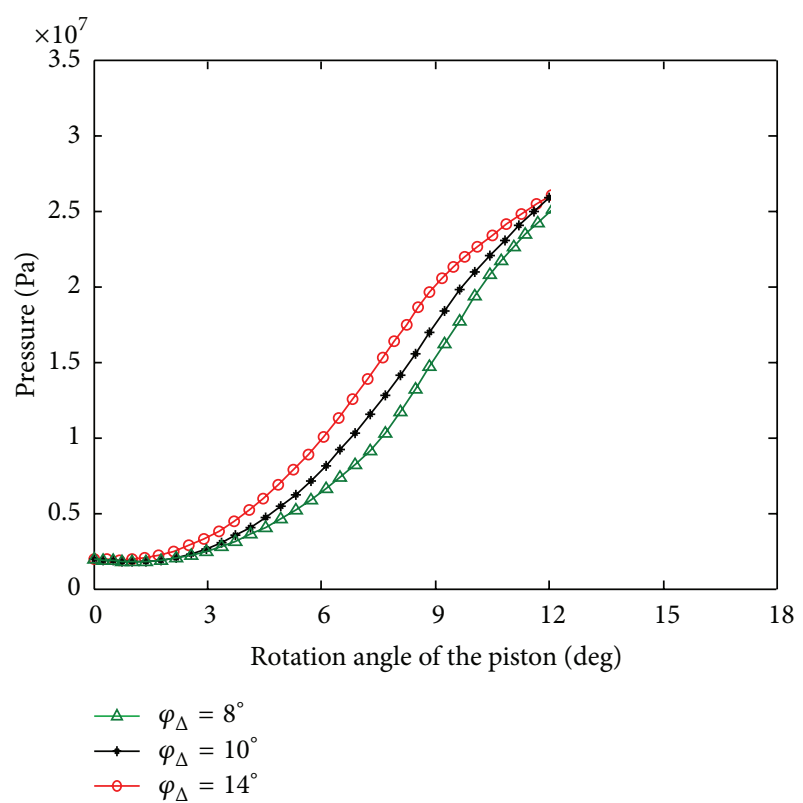

Figure 7: Pressure variation of the piston chamber.

oil shock when the piston moves to the discharge port under the original parameters of the valve plate; on the other hand, the maximum pressure gradient is 6.5 times more than the minimum pressure gradient, which would increase the noise. The ideal dynamic pressure should be nearly steady and the pressure of the piston chamber should be up to the pressure of the discharge port when the piston moves to the discharge port.

5.2. Particle Swarm Optimization (PSO) Algorithm. PSO algorithm was proposed by Kennedy and Eberhart in 1997 [14]. The basic premise is that a group of random particles are initialized; then, in each generation, the local best point of each particle is obtained by comparing the fitness value with early generations and the global best point is obtained compared with each local best point; all particles update their velocities and locations based on (15)

$$
\begin{gathered}
v_{i}^{d+1}=v_{i}^{d}+c_{1} \operatorname{rand}_{1}^{d}\left(\text { Pbest }_{i}^{d}-x_{i}^{d}\right)+c_{2} \operatorname{rand}_{2}^{d}\left(\text { Gbest }^{d}-x_{i}^{d}\right) \\
x_{i}^{d+1}=x_{i}^{d}+v_{i}^{d+1},
\end{gathered}
$$

where $v_{i}^{d}$ is the velocity of particle $i$ in generation $d, x_{i}^{d}$ is the location of particle $i$ in generation $d, c_{1}$, and $c_{2}$ are learning factors, $\operatorname{rand}_{1}^{d}, \operatorname{rand}_{2}^{d}$ are random within $[0,1]$, Pbest $_{i}^{d}$ is the local best point of particle $i$ in generation $d$, and Gbest ${ }^{d}$ is the global best point in generation $d$.

5.3. Parameter Optimization of the Valve Plate Based on PSO. The optimal objective is to avoid the oil shock and make the pressure gradient steady. As we know, the oil shock could 


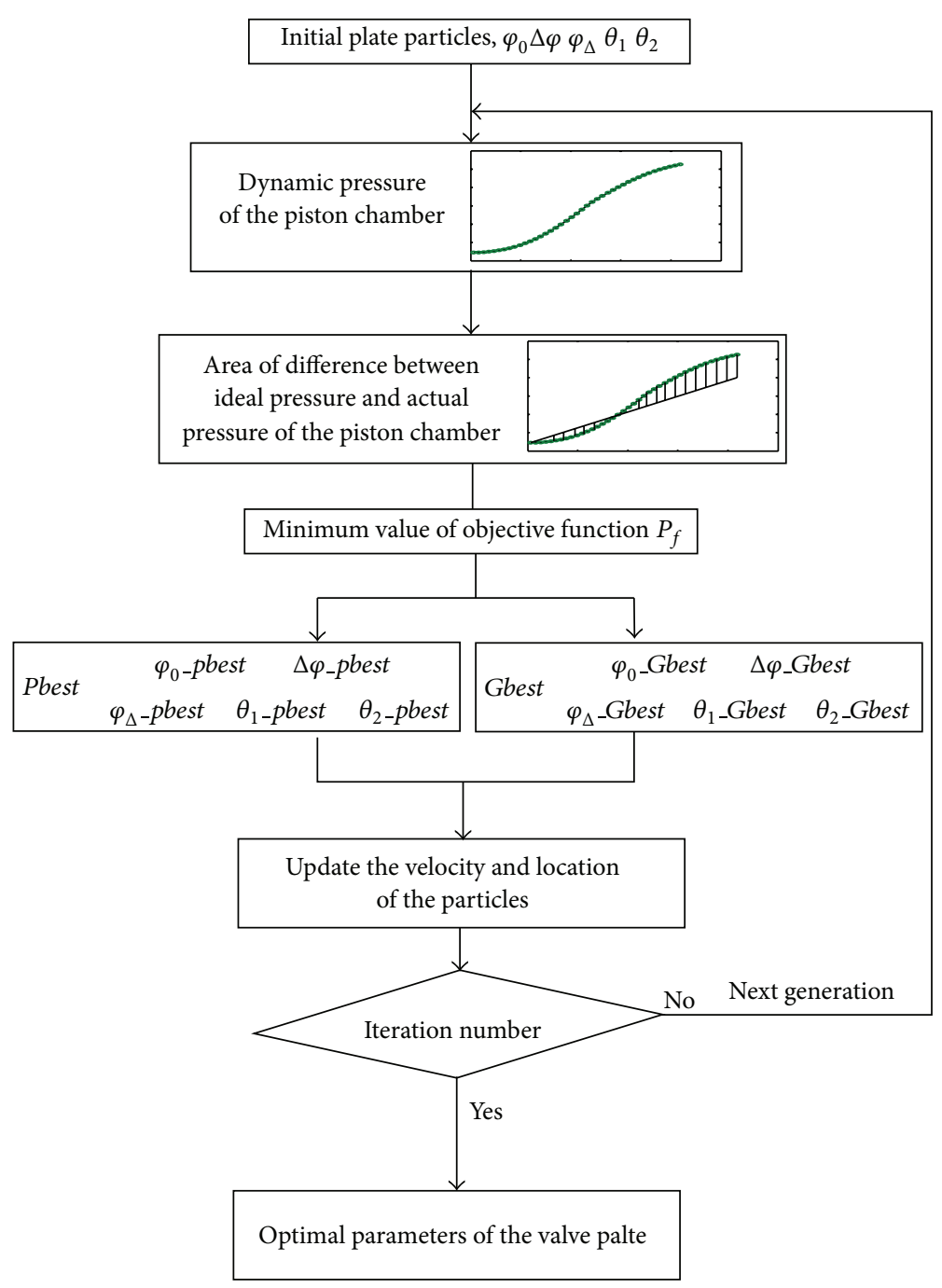

Figure 8: Flowchart of the PSO.

be avoided based on (12); meanwhile, to make the pressure gradient steady, the objective function is

$$
P_{f}=\int_{0}^{\varphi_{0}}\left|\frac{P_{s}-P_{0}}{\varphi_{0}} \varphi+P_{0}-P\right| \mathrm{d} \varphi,
$$

where $P_{f}$ is the objective function, $P_{s}$ is the pressure of the discharge port, $P_{0}$ is the initial pressure of the piston chamber when the piston moves out from the suction port, $\varphi_{0}$ is the close angle, and $P$ is the pressure variation of the piston chamber.

As we can see from (16), the integral of difference between the ideal pressure and actual pressure of the piston chamber is the area of the two pressure curves in the close angle, and the pressure gradient could be nearly steady. The close angle $\varphi_{0}$, cross angle $\Delta \varphi$, wrap angle of $\mathrm{V}$-groove $\varphi_{\Delta}$, opening angle of $\mathrm{V}$-groove $\theta_{1}$, and vertex angle $\theta_{2}$ should be optimized.

Figure 8 shows the flow chart of the parameter optimization based on the PSO.

The pressure of the piston chamber under original parameters and optimal parameters based on PSO is shown in Figure 9.
As we can see from Figure 9, the ideal pressure of the piston is steady from the initial pressure to the pressure of the discharge port. The close angle $\varphi_{0}$ is $12.46^{\circ}$, cross angle $\Delta \varphi$ is $3.4^{\circ}$, wrap angle of $\mathrm{V}$-groove $\varphi_{\Delta}$ is $13.58^{\circ}$, opening angle of $\mathrm{V}$-groove $\theta_{1}$ is $25.34^{\circ}$, and vertex angle $\theta_{2}$ is $78.52^{\circ}$. The maximum pressure gradient is only 1.9 times more than the minimum pressure gradient after optimization, which decreases by $70.8 \%$ compared to the original parameters.

5.4. Comparison of Flow Fluctuation before and after Optimization. Flow fluctuation should be calculated after optimization, and the variation process of the pump flow is shown in Figure 10.

Stage 1. The V-groove is surrounded fully by the first piston chamber

$$
\begin{aligned}
Q_{1}= & A_{Z} \omega R \tan \gamma\left[\frac{\cos (\varphi-0.5 \alpha)}{2 \sin 0.5 \alpha}-\sin \varphi\right] \\
& -\operatorname{sign}\left(p_{s}-p\right) C_{d} A_{0} \sqrt{\frac{2\left|p_{s}-p\right|}{\rho}},
\end{aligned}
$$




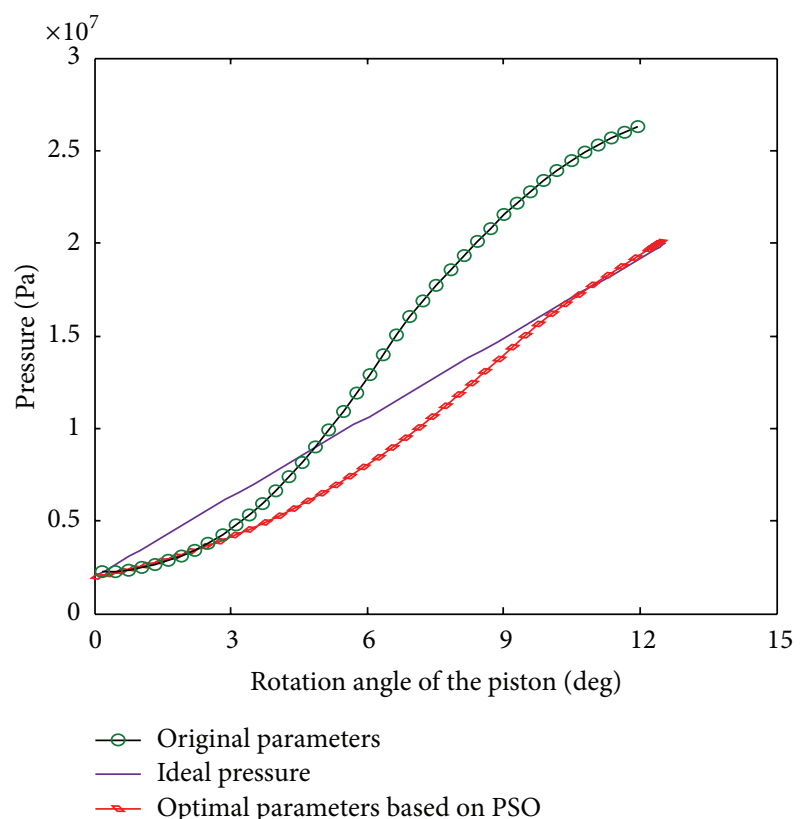

FIgURE 9: Dynamic pressure before and after optimization.

$$
\begin{aligned}
\frac{d P}{d \varphi}= & E \frac{C_{d} A_{0} \sqrt{\left(2\left(P_{s}-P\right) / \rho\right)}-k_{l} P}{\omega V_{0}} \\
& +E \frac{A \omega R \tan \gamma \sin \left(\Delta \varphi-\left(\varphi_{0} / 2\right)+\varphi\right)}{\omega V_{0}}, \\
\frac{d P}{d \varphi}= & E \frac{-C_{d} A_{0} \sqrt{\left(2\left(P-P_{s}\right) / \rho\right)}-k_{l} P}{\omega V_{0}} \\
& +E \frac{A \omega R \tan \gamma \sin \left(\Delta \varphi-\left(\varphi_{0} / 2\right)+\varphi\right)}{\omega V_{0}} .
\end{aligned}
$$

Stage 2. The fifth piston chamber departs from the discharge port from the end of Stage 1

$$
Q_{2}=A_{Z} \omega R \tan \gamma\left[\frac{\cos (\varphi-0.5 \alpha)}{2 \sin 0.5 \alpha}\right] .
$$

Stage 3. The first piston chamber moves to the $\alpha$ from the end of Stage 2

$$
Q_{3}=A_{Z} \omega R \tan \gamma\left[\frac{\cos (\varphi-0.5 \alpha)}{2 \sin 0.5 \alpha}-\sin (\varphi+8 \alpha)\right] .
$$

Stage 4. The first piston chamber moves to $2 \alpha$ from the end of Stage 3

$$
Q_{4}=A_{Z} \omega R \tan \gamma \frac{\cos (\varphi-1.5 \alpha)}{2 \sin 0.5 \alpha},
$$

where $\alpha=\pi / Z, Z$ is the number of pistons.

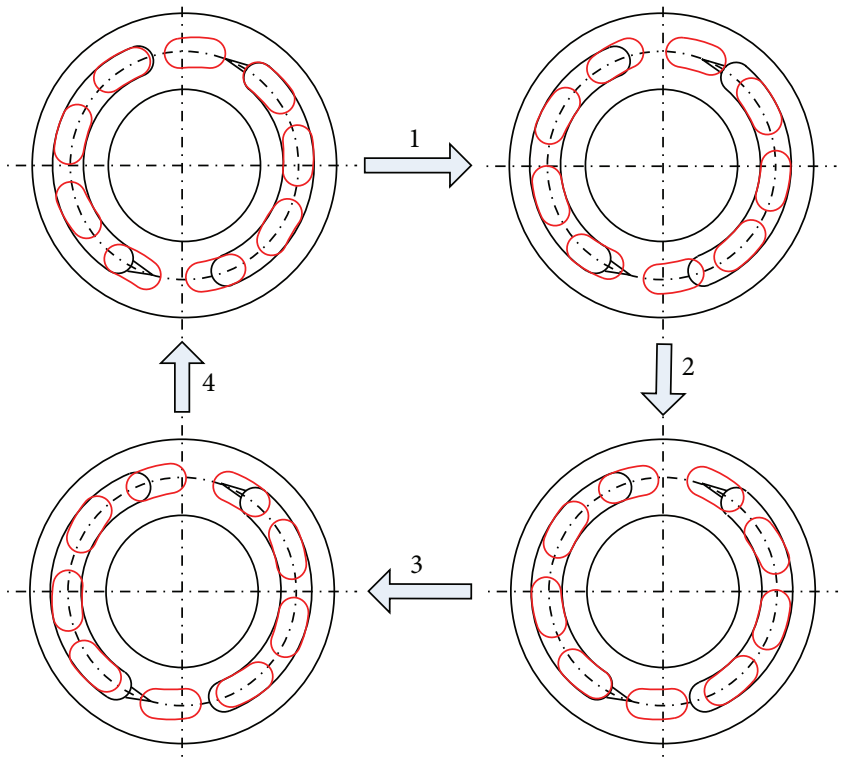

FIGURE 10: The variation process of pump flow.

Figure 11 shows the flow fluctuation between original parameters and optimized parameters. The flow fluctuation can be calculated by (21)

$$
\delta_{\mathrm{Q}}=\frac{Q_{t \max }-Q_{t \min }}{Q_{t p}},
$$

where $\delta_{Q}$ is the flow fluctuation, $Q_{t \text { max }}$ is the maximum flow value, $Q_{t \text { min }}$ is the minimum flow value, and $Q_{t p}$ is the mean flow value.

As we can see from Figure 11, the flow fluctuation is $4.67 \%$ under the original parameters; in contrast, the flow fluctuation is $3.67 \%$ under the optimized parameters, which declines by $21.4 \%$. The effectiveness of the parameters optimization of the valve plate can be concluded.

\section{Experiments}

The analysis results obtained from this paper are verified by real pump flow fluctuation experiments. The verification experiment devices are illustrated in Figure 12.

Before optimization, experiment with original valve plate is carried out and results are showed as follows.

It can be seen from Figure 13 that the flow fluctuation is $5.21 \%$ under the original parameters. Then, the design parameters of valve plate are optimized, and the experiment results with optimized valve plate are shown in Figure 14.

It can be seen from Figure 14 that the flow fluctuation is $4.22 \%$ under the optimized parameters, which declines by $19 \%$ compared with original valve plate. Although flow fluctuation decreasing effect is less than the value of the theoretical analysis due to machining errors, the optimization method proposed in this paper is proven to be effective and feasible in hydraulic pump engineering design. 


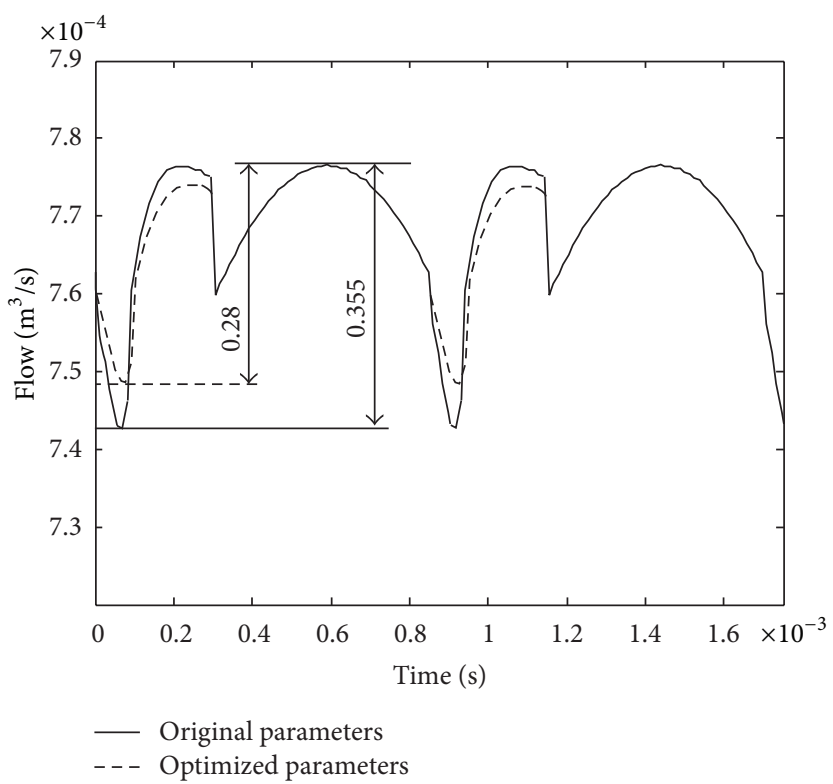

FIgURE 11: Flow fluctuation before and after optimization.
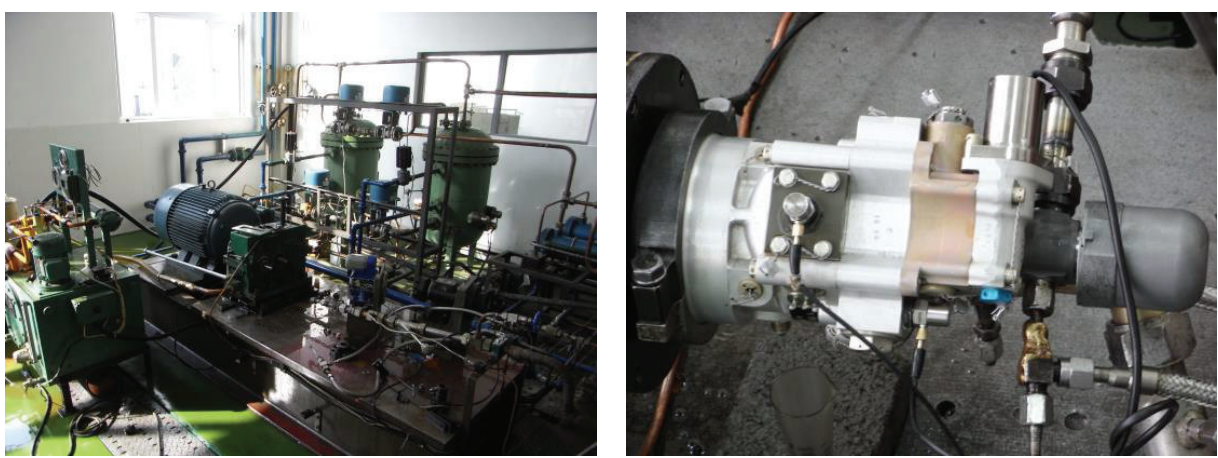

(a) Pump flow fluctuation experiments devices

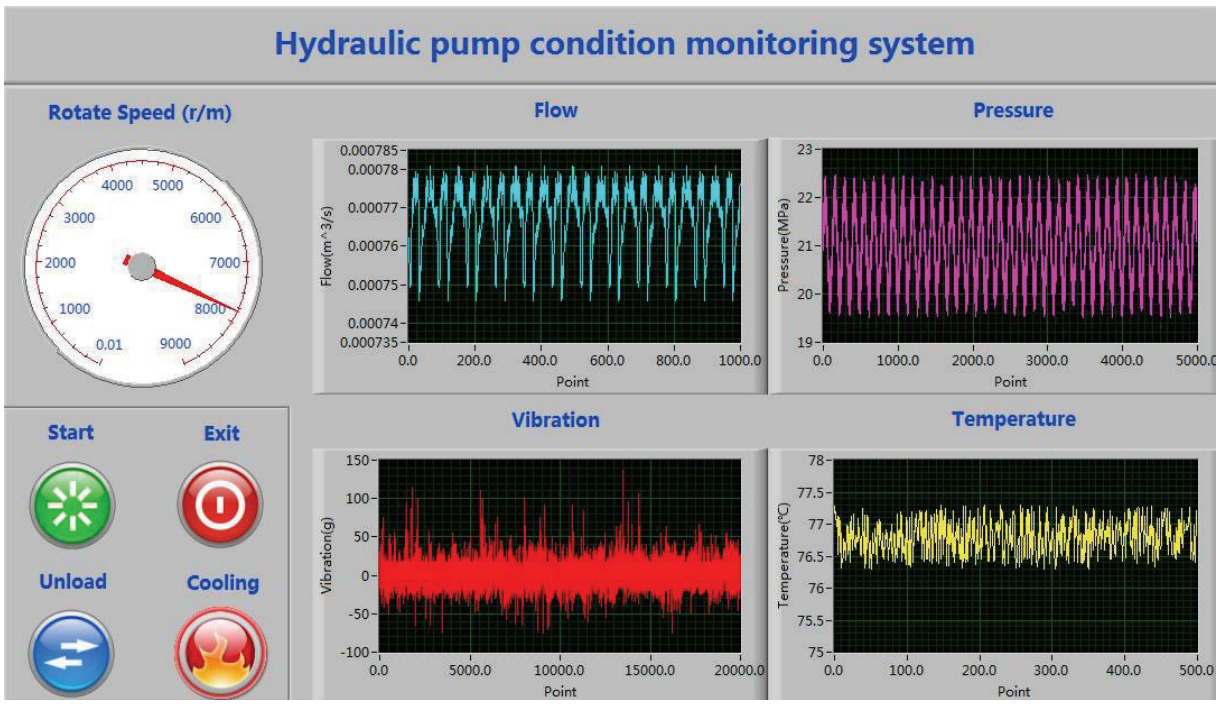

(b) Data collection software

FIgURE 12: Pump flow fluctuation experiments devices. 


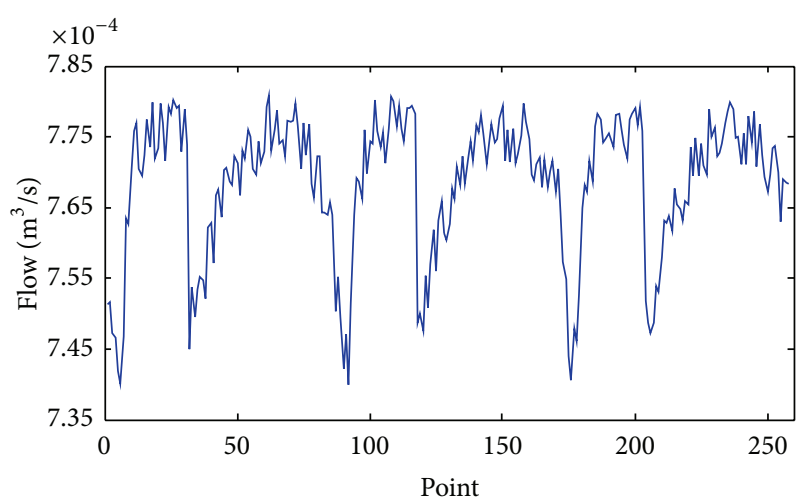

FIGURE 13: Experiment with original valve plate.

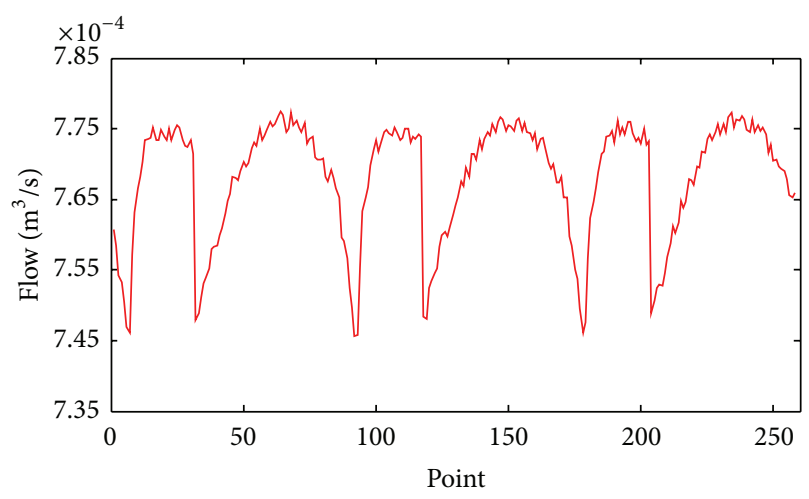

(a) Experiment with optimized valve plate

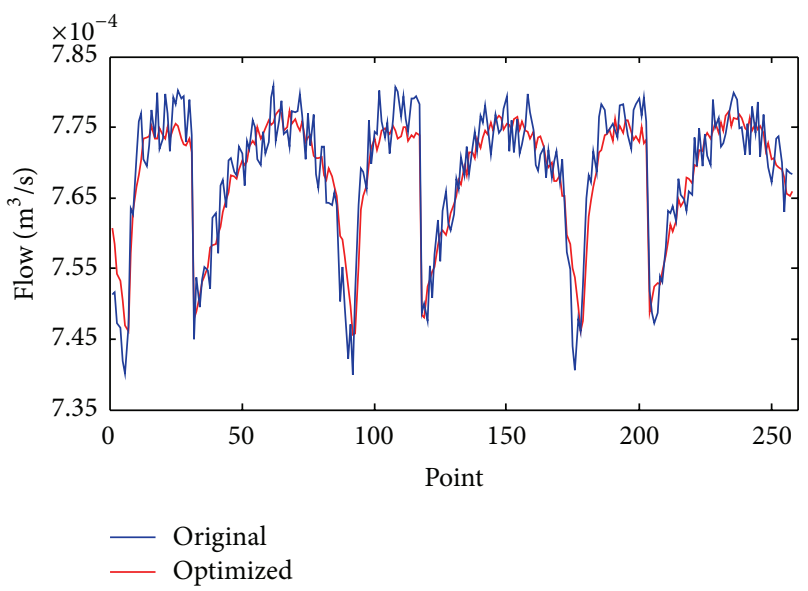

(b) Comparison between original and optimized results

FIGURE 14: Experiment with optimized valve plate.

\section{Conclusion}

(1) The oil shock can be avoided under the relation between $\Delta \varphi$ and $\varphi_{0}$; then, the effect of the close angle $\varphi_{0}$, cross angle $\Delta \varphi$, wrap angle of $\mathrm{V}$-groove $\varphi_{\Delta}$, opening angle of $\mathrm{V}$-groove $\theta_{1}$, and vertex angle $\theta_{2}$ on the pressure gradient of the piston chamber is analyzed.

(2) After optimization based on the PSO, the close angle $\varphi_{0}$ is $12.46^{\circ}$, cross angle $\Delta \varphi$ is $3.4^{\circ}$, wrap angle of $\mathrm{V}$-groove $\varphi_{\Delta}$ is $13.58^{\circ}$, opening angle of $\mathrm{V}$-groove $\theta_{1}$ is $25.34^{\circ}$, and vertex angle $\theta_{2}$ is $78.52^{\circ}$.

(3) The maximum pressure gradient is only 1.9 times more than the minimum pressure gradient after optimization, which decreases by $70.8 \%$ compared to the original parameters. On the other hand, the flow fluctuation decreases by $21.4 \%$ after the optimization.

\section{Conflict of Interests}

The authors declare that there is no conflict of interests regarding the publication of this paper.

\section{Acknowledgments}

The authors would like to appreciate the support of National Basic Research Program of China (973 Program) (2014CB046402) and National Natural Science Foundation of China (51175014 and 51305011).

\section{References}

[1] H. Yang, J. Ma, and B. Xu, "Research status of axial piston pump fluid-borne noise," Journal of Mechanical Engineering, vol. 45, no. 8, pp. 71-79, 2009.

[2] W. Kemmetmüller, F. Fuchshumer, and A. Kugi, "Nonlinear pressure control of self-supplied variable displacement axial piston pumps," Control Engineering Practice, vol. 18, no. 1, pp. 84-93, 2010.

[3] N. D. Manring and F. A. Damtew, "The control torque on the swash plate of an axial-piston pump utilizing piston-bore springs," Journal of Dynamic Systems, Measurement and Control, vol. 123, no. 3, pp. 471-478, 2001.

[4] J. M. Bergada, J. Watton, and S. Kumar, "Pressure, flow, force, and torque between the barrel and port plate in an axial piston pump," Journal of Dynamic Systems, Measurement and Control, vol. 130, no. 1, Article ID 011011, 16 pages, 2008.

[5] N. D. Manring and V. S. Mehta, "Physical limitations for the bandwidth frequency of a pressure controlled, axial-piston pump," Journal of Dynamic Systems, Measurement and Control, vol. 133, no. 6, Article ID 061005, 2011.

[6] J. M. Bergada, S. Kumar, D. L. Davies, and J. Watton, "A complete analysis of axial piston pump leakage and output flow ripples," Applied Mathematical Modelling, vol. 36, no. 4, pp. 1731-1751, 2012.

[7] K. L. Xing and C. L. Na, "Influence of damping configuration on valve plate of axial piston pump on pressure characteristics," Journal of Gansu University of Technology, vol. 21, pp. 35-39, 1995.

[8] J.-e. Ma, Y.-t. Fang, B. Xu, and H.-Y. Yang, "Optimization of cross angle based on the pumping dynamics model," Journal of Zhejiang University A, vol. 11, no. 3, pp. 181-190, 2010.

[9] C. Guan and Z. Jiao, "Dynamic optimization method for valve plate structure of aviation piston pump," Journal of Beijing University of Aeronautics and Astronautics, vol. 37, no. 3, pp. 274-278, 2011.

[10] N. D. Manring, "Valve-plate design for an axial piston pump operating at low displacements," Journal of Mechanical Design, vol. 125, no. 1, pp. 200-205, 2003.

[11] A. D. Potolea, L. Vaida, and C. Vaida, "Automated adaptive adjustment of the distribution ports for axial piston pumps," in 
Proceedings of the IEEE International Conference on Automation, Quality and Testing, Robotics (AQTR'06), pp. 288-291, rom, May 2006.

[12] A. Johansson, J. Ölvander, and J.-O. Palmberg, "Experimental verification of cross-angle for noise reduction in hydraulic piston pumps," Journal of Systems and Control Engineering, vol. 221, no. 3, pp. 321-330, 2007.

[13] N. Chenglie and W. Jingxiu, "Optimal design of noiseproof valve plate of axial piston pump with variable displatement and single-or double-staged constant pressure," Journal of Gansu University of Technology, vol. 11, pp. 46-57, 1985.

[14] J. Kennedy and R. C. Eberhart, "Discrete binary version of the particle swarm algorithm," in Proceedings of the IEEE International Conference on Systems, Man, and Cybernetics, pp. 41044108, Orlando, Fla, USA, October 1997. 


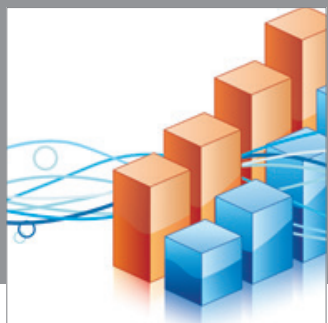

Advances in

Operations Research

mansans

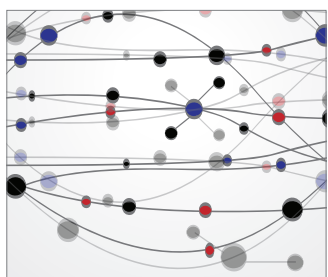

The Scientific World Journal
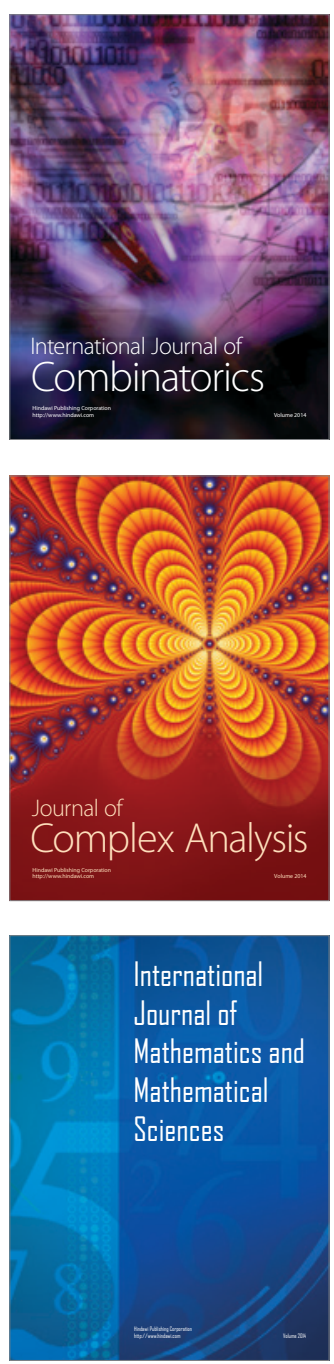
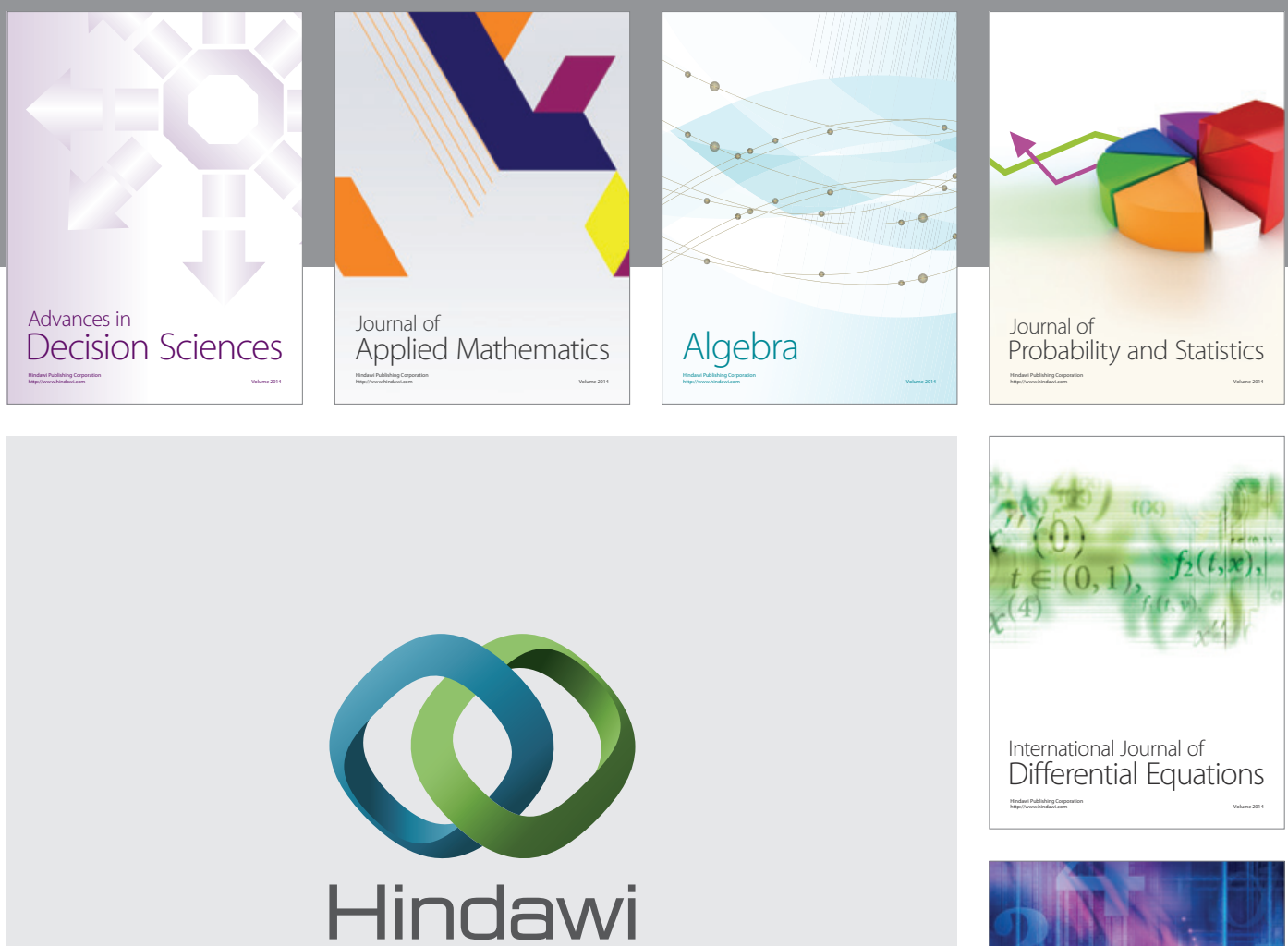

Submit your manuscripts at http://www.hindawi.com
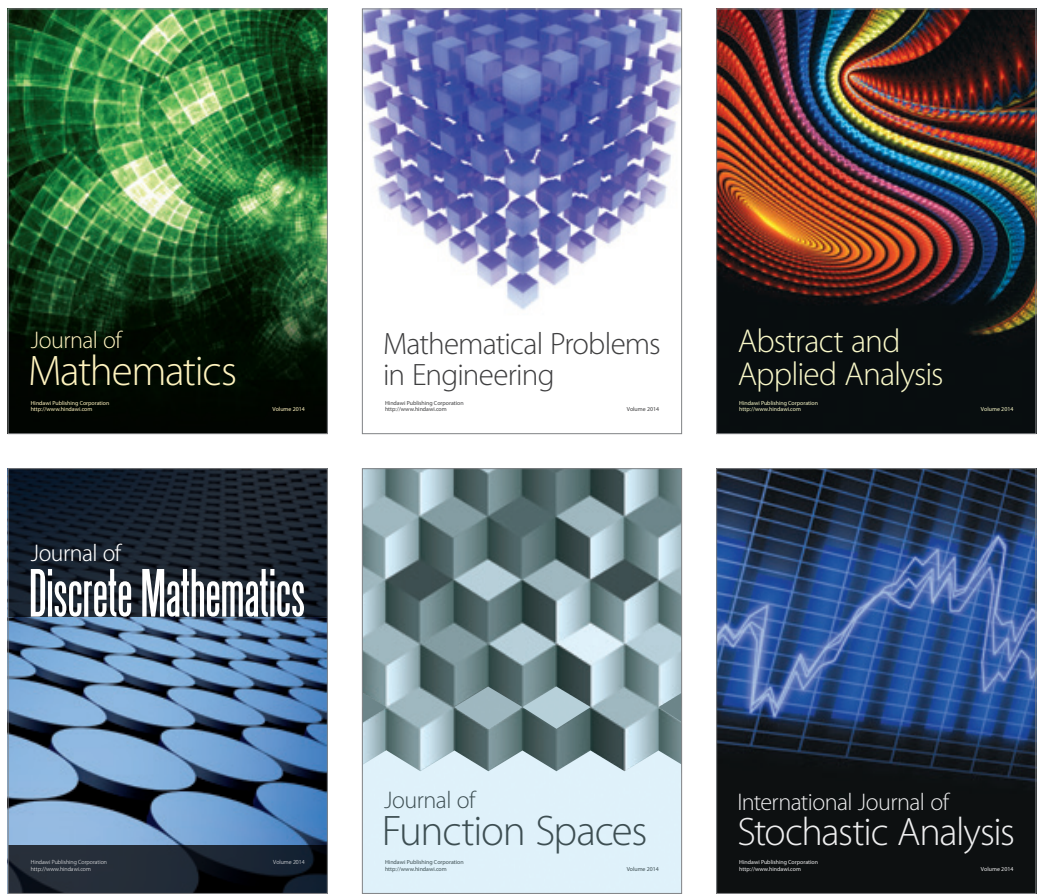

Journal of

Function Spaces

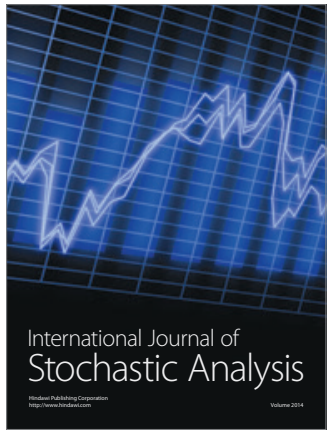

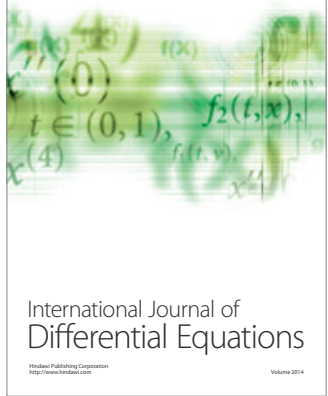
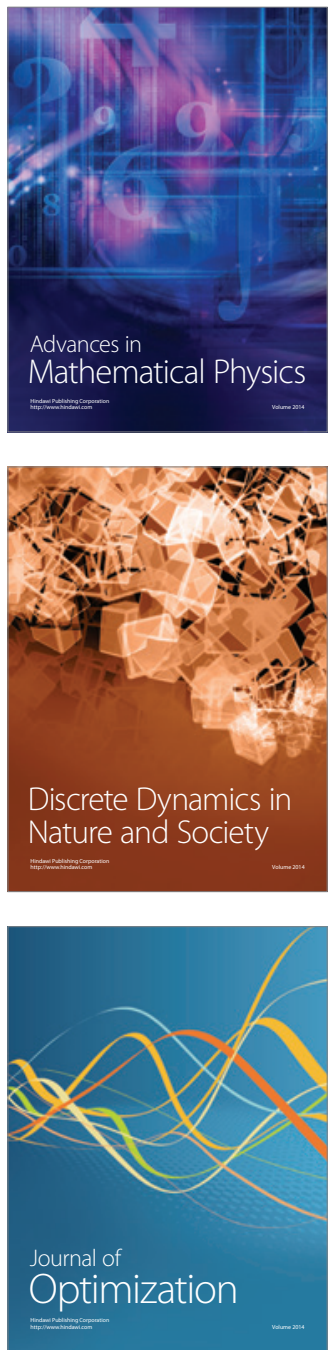